\title{
Structural Characterization and Ultraviolet Photoresponse of GaN Nanodots Grown by Molecular Beam Epitaxy
}

\author{
Mahesh Kumar ${ }^{1,2}$, Basanta Roul ${ }^{1,2}$, Thirumaleshwara N. Bhat ${ }^{1}$, Mohana K. Rajpalke ${ }^{1}$, and S. B. Krupanidhi ${ }^{1 *}$ \\ ${ }^{1}$ Materials Research Centre, Indian Institute of Science, Bangalore 560012, India \\ ${ }^{2}$ Central Research Laboratory, Bharat Electronics, Bangalore 560013, India
}

Received June 16, 2012; accepted July 7, 2012; published online July 27, 2012

The present work explores the electrical transport and UV photoresponse properties of GaN nanodots (NDs) grown by molecular beam epitaxy (MBE). Single-crystalline wurtzite structure of GaN NDs is verified by X-ray diffraction and transmission electron microscopy (TEM). The interdigitated electrode pattern was created and current-voltage (I-V) characteristics of GaN NDs were studied in a metal-semiconductor-metal configuration. Dark $I-V$ characteristics of lateral grown GaN NDs obeyed the Frenkel-Poole emission model, and the UV response of the device was stable and reproducible with on/off. The responsivity of the detectors is found to be $330 \mathrm{~A} / \mathrm{W}$ with an external quantum efficiency of $1100 \%$. (C) 2012 The Japan Society of Applied Physics

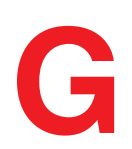
roup III-nitride materials have been extensively investigated due to their applications in lightemitting diodes, laser diodes, and photodetectors (PDs). UV PDs are important devices that can be used in various civilian as well as military applications such as space communications, ozone-layer monitoring, and flame detection. The wide direct bandgap and excellent chemical and thermal stabilities of $\mathrm{GaN}$ make it particularly suitable for extreme environmental applications of UV PDs. ${ }^{1,2)}$ Highquality GaN epitaxial films have been grown by molecular beam epitaxy (MBE) and metal organic chemical vapor deposition (MOCVD). Using these epitaxial films, various types of GaN UV detector have been demonstrated. ${ }^{3-5)}$ However, the performance of GaN-based UV detectors is limited by the high-density threading dislocation of $\mathrm{GaN}$, which has been proved to be the main path for the reversebias leakage of GaN-based devices. ${ }^{6}$ ) Other than GaN films, it is also possible to grow and use one-dimensional (1D) $\mathrm{GaN}$ nanowires (NWs) and zero-dimensional (0D) GaN nanodots (NDs) for UV detectors. 1D and OD semiconductor nanostructures have attracted much attention recently due to their interesting size-dependent electrical, optical, and transport properties. Recently, Weng et al. have reported the fabrication of GaN NW UV PDs. ${ }^{7)}$ It was found that GaN NW PDs can provide a higher responsivity and a larger UV-to-visible rejection ratio than the conventional GaNfilms-based PDs. There are some reports on the fabrication of GaN NW PDs ${ }^{8,9)}$ but not much on GaN ND PDs in the literature. In the present study, GaN NDs were grown on semi-insulating $\mathrm{Si}$ substrates and their transport and UV photoresponse properties were studied.

The GaN ND samples were grown using a plasma-assisted MBE (PAMBE) system. The semi-insulating Si(111) substrates (resistivity : $>3000 \Omega \mathrm{cm}$ ) were chemically cleaned followed by dipping in $5 \% \mathrm{HF}$ to remove the surface oxide. The GaN NDs were grown by a two step-growth method. The initial low-temperature nucleation layer was deposited at $600^{\circ} \mathrm{C}$ for $5 \mathrm{~min}$. Further, the substrate temperature was raised to $750{ }^{\circ} \mathrm{C}$ to fabricate the NDs. The duration of nanodot growth was $2 \mathrm{~h}$. The Ga effusion cell temperature, nitrogen flow rate, and plasma power were kept at $880^{\circ} \mathrm{C}, 1 \mathrm{sccm}$, and $350 \mathrm{~W}$, respectively. The surface morphologies of GaN NDs were investigated in ultrahigh vacuum using needle a sensor

*E-mail address: sbk@mrc.iisc.ernet.in

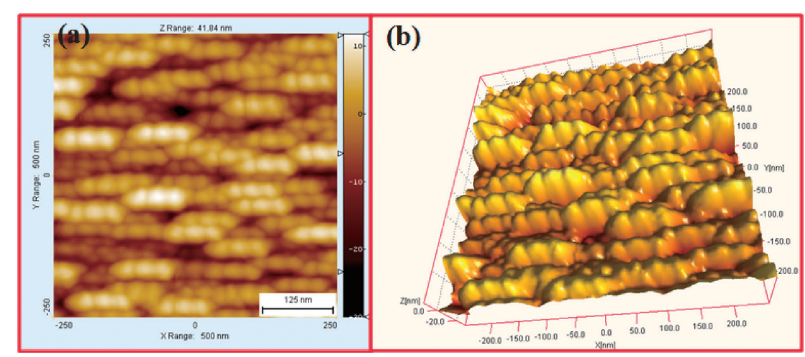

Fig. 1. (a) Planar and (b) 3D views of GaN NDs.

by atomic force microscopy (AFM), which was supplied by Omicron Nanotechnology, Germany. Structural and optical properties of GaN NDs were investigated by powder X-ray diffraction (XRD) and photoluminescence (PL) measurements. The single-crystalline structure of GaN NDs is verified by transmission electron microscopy (TEM). The interdigited electrode pattern was created on GaN NDs by using photolithography and lift-off techniques. The electrodes were formed by thermal evaporation of $\mathrm{Al}$ (thickness. $\sim 150 \mathrm{~nm}$ ). The distance between the two fingers was $10 \mu \mathrm{m}$ and the width of each finger was $10 \mu \mathrm{m}$. Electrical measurements and photoresponse were measured in air using a Keithley 236. Current-voltage $(I-V)$ characteristics of the devices were measured in dark and under UV illumination. The UV source consists of a handheld lamp of $360 \mathrm{~nm}$ wavelength and $0.3 \mathrm{~mW} / \mathrm{cm}^{2}$ intensity.

The size and density distribution of GaN NDs were determined by AFM. Figures 1(a) and 1(b) show planar and 3D AFM images of GaN NDs, respectively, and it can be seen that GaN NDs are uniformly grown over the substrate. The average size of GaN NDs was around $\sim 25 \mathrm{~nm}$ with standard deviation of $0.22 \mathrm{~nm}$ and density of $\sim(1.1 \pm 2) \times$ $10^{11} \mathrm{~cm}^{-2}$ calculated from the AFM image. Further detailed structural information on the GaN ND is obtained by TEM analysis. The GaN NDs were removed from the substrate by sonication in acetone and placed on TEM grids. Figure 2(a) shows typical bright-field TEM images of GaN ND, and the size of the ND is close to the average size, which is calculated from the AFM image. The corresponding highresolution TEM (HRTEM) image of GaN ND is shown in Fig. 2(b). The HRTEM shows one of the corner edges of an $\mathrm{ND}$ and the interplanar spacing, as observed from the fringe pattern of the HRTEM image, is $0.258 \mathrm{~nm}$, which corre- 


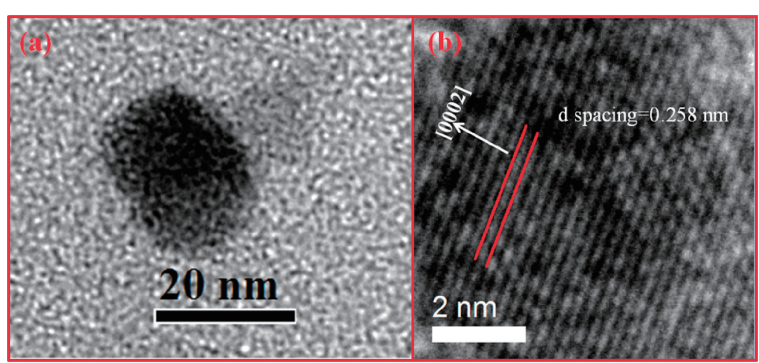

Fig. 2. (a) Typical TEM image of a GaN ND and (b) HRTEM image of the corner edge of the ND.
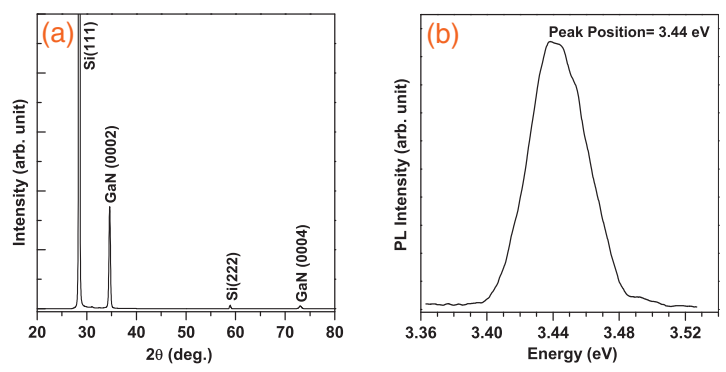

Fig. 3. (a) XRD $2 \theta / \omega$ scan and (b) room temperature PL spectra of the GaN NDs grown on $\mathrm{Si}(111)$ substrate.

sponds to the (0002) lattice spacing of fully relaxed GaN layers. ${ }^{10)}$ These results clearly demonstrate that the as-grown NDs are fairly single crystalline, and are crystallized hexagonally along the [0001] direction with uniform geometry. Figure 3(a) shows XRD 2 $\theta / \omega$ scan of GaN NDs grown on $\mathrm{Si}(111)$ substrate. From the figure, it can be seen that except the substrate peaks, only a (0002) GaN diffracted peak at $2 \theta=34.65^{\circ}$ is present, indicating that the GaN NDs are highly oriented along the [0001] direction of the wurtzite structures. The optical properties of GaN NDs were characterized by PL measurements using a $\mathrm{He}-\mathrm{Cd}$ laser $(30 \mathrm{~mW}$, $325 \mathrm{~nm}$ ) as the excitation source at room temperature and shown in Fig. 3(b). The GaN NDs show a strong band-edge emission peak at $3.44 \mathrm{eV}$.

The schematic diagram of the device structure is shown in Fig. 4(a), while Figs. 4(b) and 4(c) show the optical microscopy and SEM images of the device, respectively. Figure 5(a) shows dark $I-V$ characteristics of GaN NDs at room temperature. The $I-V$ characteristic has been analyzed in terms of the Frenkel-Poole emission model. Based on this model, the current is given by ${ }^{11,12)}$

$$
I \propto E_{\mathrm{b}} \exp \left[-\frac{q\left(\phi_{\mathrm{t}}-\sqrt{q E_{\mathrm{b}} / \pi \varepsilon_{0} \varepsilon_{\mathrm{s}}}\right)}{k_{\mathrm{B}} T}\right],
$$

where $E_{\mathrm{b}}$ is the applied electric field, $\phi_{\mathrm{t}}$ is the barrier height for electron emission from the trap state, $\varepsilon_{0}$ is the permittivity of free space, $\varepsilon_{\mathrm{s}}$ is the relative dielectric permittivity of the GaN material, $k_{\mathrm{B}}$ is Boltzmann's constant, and $T$ is the temperature. By rearranging eq. $(1), \log \left(I / E_{\mathrm{b}}\right)$ should be a linear function of $E_{\mathrm{b}}{ }^{1 / 2}$ for current transport by FrenkelPoole emission, i.e.,

$$
\log \left(\frac{I}{E_{\mathrm{b}}}\right)=\frac{q}{k_{\mathrm{B}} T} \sqrt{\frac{q E_{\mathrm{b}}}{\pi \varepsilon_{0} \varepsilon_{\mathrm{s}}}}-\frac{q \phi_{\mathrm{t}}}{k_{\mathrm{B}} T}+\log C
$$

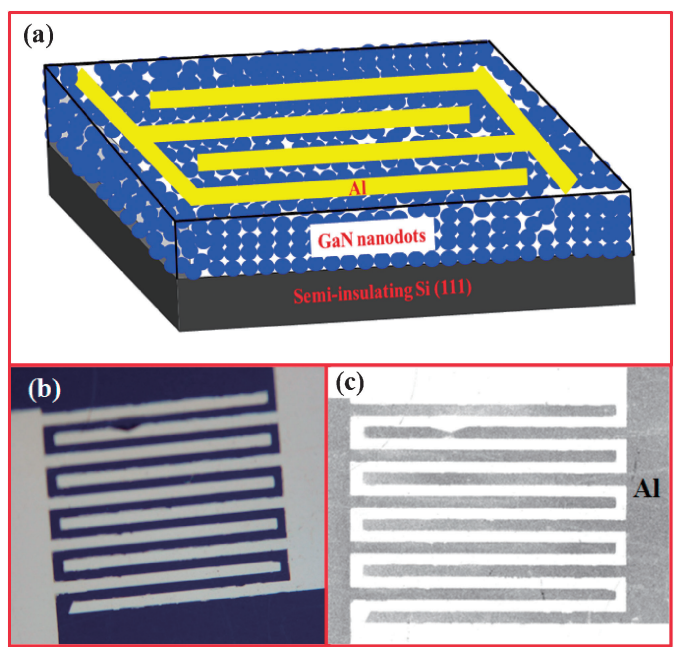

Fig. 4. (a) Shows the schematic diagram of the device structure (not to scale), (b) and (c) show the optical microscopy and SEM images of the device, respectively.
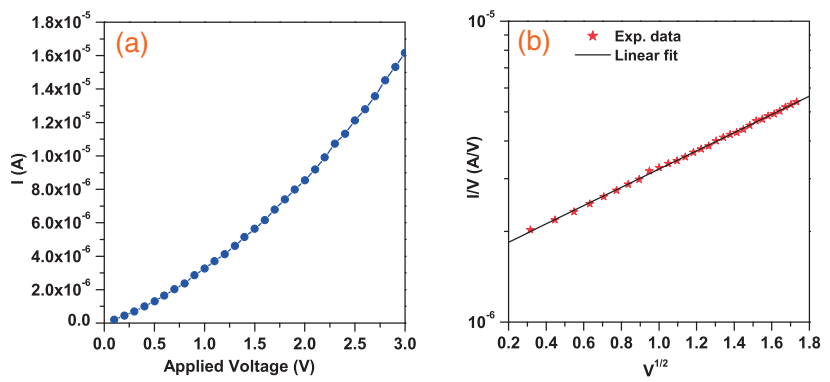

Fig. 5. (a) Dark $I-V$ characteristics of device at room temperature and (b) current $I$ divided by the applied bias $V$ vs square root of $V$ for GaN NDs detector.

where $C$ is a constant. Assuming a linear dependence of $E_{\mathrm{b}}$ with voltage $(V), \log (I / V)$ should be a linear relationship with $V^{1 / 2}$. Figure 5(b) shows $\log (I / V)$ as a function of $V^{1 / 2}$, which follows the expected evolution predicted using eq. (2), which means that conduction in the present case obeys the Frenkel-Poole emission from the traps. A similar type of behavior was observed in GaN films ${ }^{13)}$ and InAs/InGaAs/ GaAs quantum dot structures. ${ }^{14)}$

In Fig. 6(a) shows the typical $I-V$ characteristics in the dark (squares) and under UV illumination (circles) of the device at $360 \mathrm{~nm}$ with $0.3 \mathrm{~mW} / \mathrm{cm}^{2}$. Figure $6(\mathrm{~b})$ shows the measured transient response of the fabricated device at $3 \mathrm{~V}$, as we switched the UV excitation on and off. From the figure, it was found that the dynamic response of the device was stable and reproducible with an on/off current contrast ratio of around 10. It was also found that the photocurrent increased rapidly initially and then increased much slower as we turned on the UV excitation. A similar slow response was also observed as we turned off the UV light. A similar type of behavior observed in $\mathrm{GaN}^{7)}$ and $\mathrm{ZnO}^{15)} \mathrm{NWs}$ due to the surface-defect-related trapping center could retard the speeds of charge-carrier collection upon UV illumination and charge-carrier recombination as the UV light was turned off.

Responsivity $\left(R_{\lambda}\right)$, the ratio of photocurrent generated to the intensity of the incident light on the effective area 

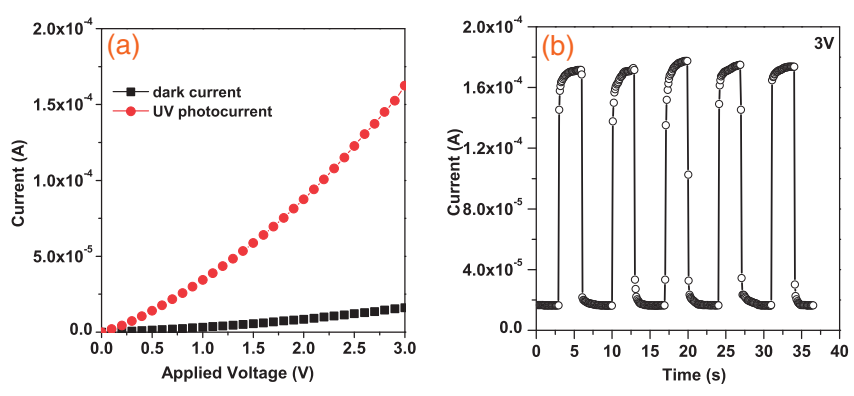

Fig. 6. (a) Typical $I-V$ characteristics in dark (squares) and under UV illumination (circles) of the device at $360 \mathrm{~nm}$ with $0.3 \mathrm{~mW} / \mathrm{cm}^{2}$.

(b) Transient response of the fabricated device at $3 \mathrm{~V}$, as we switched the UV excitation on and off.

of a photoconductor, and the external quantum efficiency $(\eta)$, defined as the number of electrons detected per incident photon, are two important parameters for the photoconductors. The higher values of $R_{\lambda}$ and $\eta$ correspond to high sensitivity. The $R_{\lambda}$ and $\eta$ were calculated as $R_{\lambda}=$ $I_{\lambda} /\left(P_{\lambda} S\right)^{16)}$ and $\eta=h c R_{\lambda} /(\mathrm{e} \lambda),{ }^{17)}$ where $I_{\lambda}$ represents the photocurrent ( $I_{\text {illumination }}-I_{\text {dark }}$ ), $P_{\lambda}$ is the light intensity, $S$ is the effective device area under illumination, and $\lambda$ is the excitation wavelength. From our experimental results, $R_{\lambda}$ and EQE of the device are $330 \mathrm{~A} / \mathrm{W}$ and $1100 \%$, respectively, for an incident wavelength of $360 \mathrm{~nm}$ at $3 \mathrm{~V}$. Such a result indicates that there exists a large photoconductive gain in the GaN ND PD compared with the GaN film and NW PD. ${ }^{7,18)}$ Thus, these results confirm that GaN NDs are a promising candidate for UV photodetectors and photoelectronic switches.

Photoconductive gain is given by $\tau / t$, where $\tau$ is the minority lifetime and $t$ is the transit time of majority carriers. The gain in photoconductive devices comes about because the recombination lifetime is much longer than the transit time. High gain and responsivity thus originate from efficient transport of one carrier combined with long-lived trapping of the carrier of the opposite type. ${ }^{19)}$ The extended trap state lifetime lowers the response time. Because of the large surface-to-volume ratio, NDs contain an extremely high density of surface states. Consequently, due to the pinning of the Fermi energy at the surface, NDs exhibit a depletion space charge layer which provides physical separation of electrons and holes and can lead to significantly enhanced photocarrier lifetime. The carrier transit time is significantly reduced due to the high mobility achievable in high-quality, defect-free single crystal NDs combined with small interelectrode distances. ${ }^{20)}$ Higher aspect ratio, higher localizes states and lower threading dislocation density in GaN NDs than the
GaN films, are the possible origin of higher responsivity and external quantum efficiency in GaN NDs photodetectors.

In conclusion, we have demonstrated UV sensing properties of GaN NDs, which were grown on semi-insulating $\mathrm{Si}(111)$ substrate by PAMBE. The average size of GaN NDs is around $\sim 25 \mathrm{~nm}$ and the density of NDs is $\sim 1.1 \times 10^{11}$ $\mathrm{cm}^{-2}$. XRD and TEM analyses show that the crystal structures of NDs are hexagonal wurtzite. The $I-V$ characteristics of GaN NDs were studied at room temperature and were interpreted by using the Frenkel-Poole emission model. It was also found that the dynamic UV response of the device was stable and reproducible with an on/off current contrast ratio of around 10 at $3 \mathrm{~V}$. The responsivity of the detectors is found to be $330 \mathrm{~A} / \mathrm{W}$ with an external quantum efficiency of $1100 \%$.

1) S. Nakamura: Science 281 (1998) 956.

2) J. W. Yang, A. Lunev, G. Simin, A. Chitnis, M. Shatalov, M. A. Khan, J. E. Van Nostrand, and R. Gaska: Appl. Phys. Lett. 76 (2000) 273.

3) G. Y. Xu, A. Salvador, W. Kim, Z. Fan, C. Lu, H. Tang, H. Morkoç, G. Smith, M. Estes, B. Goldenberg, W. Yang, and S. Krishnankutty: Appl. Phys. Lett. 71 (1997) 2154.

4) O. Katz, V. Garber, B. Meyler, G. Bahir, and J. Salzman: Appl. Phys. Lett. 80 (2002) 347.

5) J. Li, Y. Xu, T. Y. Hsiang, and W. R. Donaldson: Appl. Phys. Lett. 84 (2004) 2091.

6) W. P. Hsu, M. J. Manfra, D. V. Lang, S. Richter, S. N. G. Chu, A. M. Sergent, R. N. Kleiman, L. N. Pfeiffer, and R. J. Molnar: Appl. Phys. Lett. 78 (2001) 1685.

7) W. Y. Weng, T. J. Hsueh, S. J. Chang, S. B. Wang, H. T. Hsueh, and G. J. Huang: IEEE J. Sel. Top. Quantum Electron. 17 (2011) 996.

8) R. S. Chen, C. Y. Lu, K. H. Chen, and L. C. Chen: Appl. Phys. Lett. 95 (2009) 233119.

9) M. Kang, J. S. Lee, S. K. Kim, B. Min, K. Cho, G. T. Kim, M. Y. Sung, S. Kim, and H. S. Han: Jpn. J. Appl. Phys. 43 (2004) 6868.

10) B. Liu, Y. Bando, C. Tang, F. Xu, and D. Golberg: Appl. Phys. Lett. 87 (2005) 073106.

11) J. G. Simmons: Phys. Rev. B 155 (1967) 657.

12) X. Sun, D. Li, H. Jiang, Z. Li, H. Song, Y. Chen, and G. Miao: Appl. Phys. Lett. 98 (2011) 121117.

13) P. Kozodoy, J. P. Ibbetson, H. Marchand, P. T. Fini, S. Keller, J. S. Speck, S. P. DenBaars, and U. K. Mishra: Appl. Phys. Lett. 73 (1998) 975.

14) N. F. Hasbullah, J. P. R. David, and D. J. Mowbray: J. Appl. Phys. 109 (2011) 113111.

15) Y. W. Heo, B. S. Kang, L. C. Tien, D. P. Norton, F. Ren, J. R. La Roche, and S. J. Pearton: Appl. Phys. A 80 (2005) 497.

16) T. Ueda, Z. H. An, K. Hirakawa, and S. Komiyama: J. Appl. Phys. 103 (2008) 093109.

17) X. P. Chen, H. L. Zhu, J. F. Cai, and Z. Y. Wu: J. Appl. Phys. 102 (2007) 024505.

18) R. S. Chen, H. Y. Chen, C. Y. Lu, K. H. Chen, C. P. Chen, L. C. Chen, and Y. J. Yang: Appl. Phys. Lett. 91 (2007) 223106.

19) G. Konstantatos, I. Howard, A. Fischer, S. Hoogland, J. Clifford, E. Klem, L. Levina, and E. H. Sargent: Nature 442 (2006) 180.

20) C. Soci, A. Zhang, X. Y. Bao, H. Kim, Y. Lo, and D. Wang: J. Nanosci. Nanotechnol. 10 (2010) 1430. 